\title{
Fibrous Dysplasia of the Mandibular Arc, Implant-Prosthetic Rehabilitation after Resection and Reconstruction with Iliac Autogenous Bone Graft: A Case Report
}

\author{
Alessandro Rossi*1, Cristina Barbara Cattaneo ${ }^{1}$, Federico Bianchi ${ }^{2}$, Alberto Clivio $^{1}$ and Matteo Chiapasco ${ }^{1}$ \\ ${ }^{1}$ Unit of Oral Surgery Matteo Chiapasco) - Department of Health Sciences - Santi Paolo \& Carlo Hospital, University of Milan, Italy \\ ${ }^{2}$ Unit of Prosthodontics (Head Prof. Eugenio Romeo) - Department of Health Sciences - Santi Paolo \& Carlo Hospital, University of Milan, Italy
}

Received: June 01, 2018; Published: June 08, 2018

*Corresponding author: Alessandro Rossi, Unit of Oral Surgery, Department of Health Sciences - Santi Paolo \& Carlo Hospital, University of Milan, Italy Via Beldiletto 1 - 20142 Milan, Italy

\begin{abstract}
The reconstruction of maxillo-mandibular defects following resection for tumors with autogenous bone grafts has been demonstrated to be a reliable technique with good long-term results. Implants placed in the reconstructed areas were demonstrated to integrate normally, with success and survival rates comparable to those obtained with implants placed in native bone. The authors present an explicative case of this type of surgical and prosthetic rehabilitation.
\end{abstract}

Keywords: Fibrous Dysplasia; Bone Graft; Dental Implant; Resective Surgery

\section{Introduction}

The resective surgical treatment of benign and malignant bone neoplasms causes the loss of bones continuity, which determines a series of craniofacial functional and aesthetic alterations that are often hard to fix. In particular, teeth and alveolar bone loss can lead to serious complications, especially for what concerns chewing and swallowing. Clinical research put a lot of efforts in discovering new functional and aesthetic reconstruction methods in the last few years. Rehabilitation following the benign bone neoplasm surgical resection can be powered by free grafts, iliac crest bone grafts, or calvarial bone grafts. These reconstruction techniques allow an instant or late implant rehabilitation of the resection site. The aim of this work is to show, through a case report, which are the rehabilitation possibilities using osseointegrated implants in bone grafts after jaw resection. At first it is provided an overview about epidemiology and pathogenesis of fibrous dysplasia and then it follows the case report.

Fibrous dysplasia is a benign bone lesion, a non familiar non inflammatory congenital disorder of bones. In 1891 Von Recklinghausen [1] has been the first describing the case of a patient with a pathology condition of bones characterized by deformity and fibrosis; only in 1937 this clinical presentation was described as a syndrome by Lichtenstein. He introduced the term of Fibrous dysplasia in order to describe the bone polyostotic lesion previously known as "osteitis fibrosa disseminata". Jaffe e Lichteinstein also discovered that fibrous dysplasia may involve only one bone (monostotic type), or several bones (polyostotic type); then these lesions have been connected with endocrinopathy (Albright syndrome, Jaffe syndrome).

\section{Epidemiology and Pathogenesis}

Fibrous dysplasia is a condition that appears during childhood; it progresses during adolescence, and usually affects young people from 20 to 30 years old. It can be found in both sexes but it prevails among females. It is an uncommon disorder and it accounts for about $2.5 \%$ of all bone tumors, and for $7.5 \%$ of the benign bone neoplasms. Fibrous dysplasia is called monostotic when it involves only a single bone; this disease is monostotic in the $80 \%-94 \%$ of cases. The monostotic form is the most frequent and it is usually asymptomatic; in some cases the lesion is associated with pain coming from a bone's fracture. Fibrous dysplasia most frequently involves craniomaxillofacial bones, ribs, diathesis or metaphysic of tibia and femur. When a lesion starts from the upper jaw and later involves the adjacent bones (sphenoid bone, occipital, zygomatic) is called craniofacial fibrous dysplasia.

Polyostotic fibrous dysplasia appears during childhood, and it interests (25\% of cases) a limb in segmental way (especially the 
lower ones). The Polyostotic form is usually more severe and is characterized by multiple lesions involving several bones; it can lead to fractures and deformities that appear fairly early. The patients showing both these lesions and an endocrinopathy, McCune Albright syndromes, are characterized by areas of coetaneous pigmentation. Frequently this pathology is associated whit hypothyroidism. Fibrous dysplasia is associated less frequently with Cushing syndrome, acromegaly, hypogonadism, hypogonadism, and myxomas. Rarely fibrous dysplasia progresses in sarcoma. There's a $10 \%-27 \%$ chance that this disease interests the craniomaxillofacial bone; it may also extend to sphenoid, occipital bone and the zygomatic bone. The Polyostotic form (30\% cases) involves the craniomaxillofacial bone in $50 \%$ of cases and it is associated with endocrinopathy in $3 \%$ of cases.

\section{Pathological Anatomy}

All the Fibrous dysplasia forms present a similar histological aspect; is characterized by irregularly shaped trabeculae of woven bone in a background of moderately cellular fibrous connective tissue. The fibrous stroma gradually substitutes the bones. The bony trabecula assumes a variety of shapes that are called "Chinese character". It is not possible to identify a border between the lesion and the healthy bone; in fact lesions seldom appear in capsules. Histological speaking we can identify three different forms of fibrous dysplasia: sclerotic, cystic, mixed.

\section{Clinical Behavior}

The disease usually shows symptoms during the first 3 decades of life, but in some patients the symptoms can appear during childhood, progress throughout adolescence and stabilize over adulthood. Signs and symptoms depend on the lesion's localization. The most frequent symptoms are facial asymmetry, which is determined by bone's enlargement and tilted occlusal plane; frequently dysesthesias can appear in the distribution of the trigeminal nerve; maxillary sinus can be totally involved by fibrosis, it may result in nasal obstruction, sinus ostial obliteration, or sinusitis. Moreover orbital involvement results in dystopia, diplopia, decreased visual acuity, headaches.

The diagnosis of fibrous dysplasia is initially based on clinical examination and radiographic exams, and then it is confirmed by further histological examination. The clinical examination often identifies an enlargement of the involved bones which produces facial asymmetry. The lesion's radiographic image of monostotic fibrous dysplasia depends on the evolutions of the disease. Usually the radiographic images have been described as having a ground glass appearance because of the admixture of fibrous and osseous element. Computed tomography (CT) is necessary in detecting and defining these craniofacial lesions. The lesions can appear as cystic (lytic) in the beginning, or sclerotic and mixed lesions in the advanced stage.

\section{Treatment}

Today, the evidence based medicine suggests to apply a preservative surgical treatment to jaw alveolar process, and to the skull base. Early radical surgery was recommended for frontal bones, orbital bone, nasal, ethmoid, zygomatic and maxillary and mandibular. Functional and aesthetic reconstruction is powered by iliac crest bone grafts, calvarial bone graft, free grafts, vascular zed skin flaps, and vascular zed muscles. The treatment of this disease depends on the lesion's extension and location, on the appearance of signs and symptoms, and on facial aesthetics and function of the bone involved. There are different approaches to treat this disease. On one side, to treat small monostotic lesions an intraoral surgical approach with curettage and debridement is suggested, because this lesions is a benign bone neoplasm's and the functions and aesthetics of the jaw must be preserved and protected. Chen et al. [2] consider the preservative surgical approach as the best choice to treat maxillary and mandibular lesions because of functional and aesthetic preservation reasons. A radical surgery approach can determine teeth loss and functional disorders. When the disease is treated with a preservative surgical approach, it's important to organize periodical follow-up because frequently the lesions could relapse.

On the other side, in case of extensive lesions or relapse, a radical surgery approach with resection of the involved bone and following reconstruction with autogenous bone grafts or free grafts is necessary. In our work we will focus exclusively on the description of the surgical treatment (Resection and Reconstruction) and after that on implant rehabilitation.

\section{Materials and Methods \\ Case Report}

In 2008, a 37-year-old female patient has been invited to be observed in order to examine a suspect jaw lesion. Clinical examination showed an expansion of buckle cortical in the symphysis area; with a radiographic examination it has been possible to identify an extended, multi-lobed radiolucent lesion with undefined borders (Figure 1); the TC image showed an inhomogeneous lesion extended from the lower right canine to the lower left molar (Figures 2 \& $2 \mathrm{a})$. The lesion had already reached the bone cortical in some areas, while it had already invaded the soft tissue. So, it has been decided to execute a punch biopsy and a fibrosis dysplasia has been identified thanks to a histological analysis (Figures $3 \& 3 \mathrm{~b}$ ).

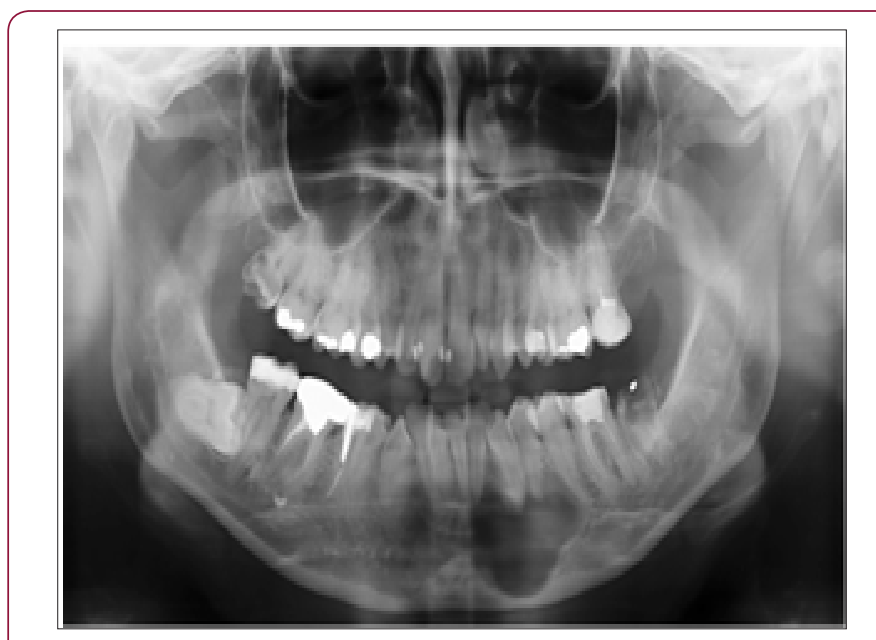

Figure 1: Radiographic examination. 


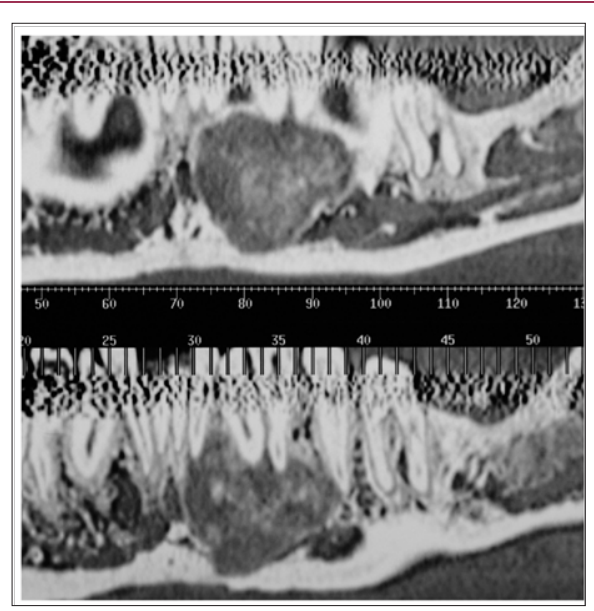

Figure 2: TC image.

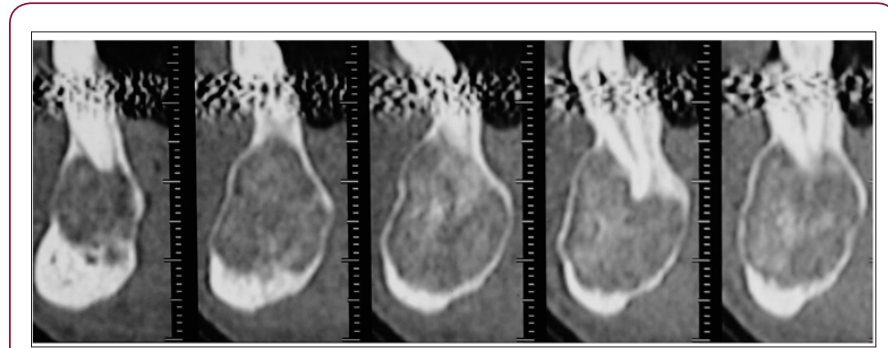

Figure 2A: TC image.

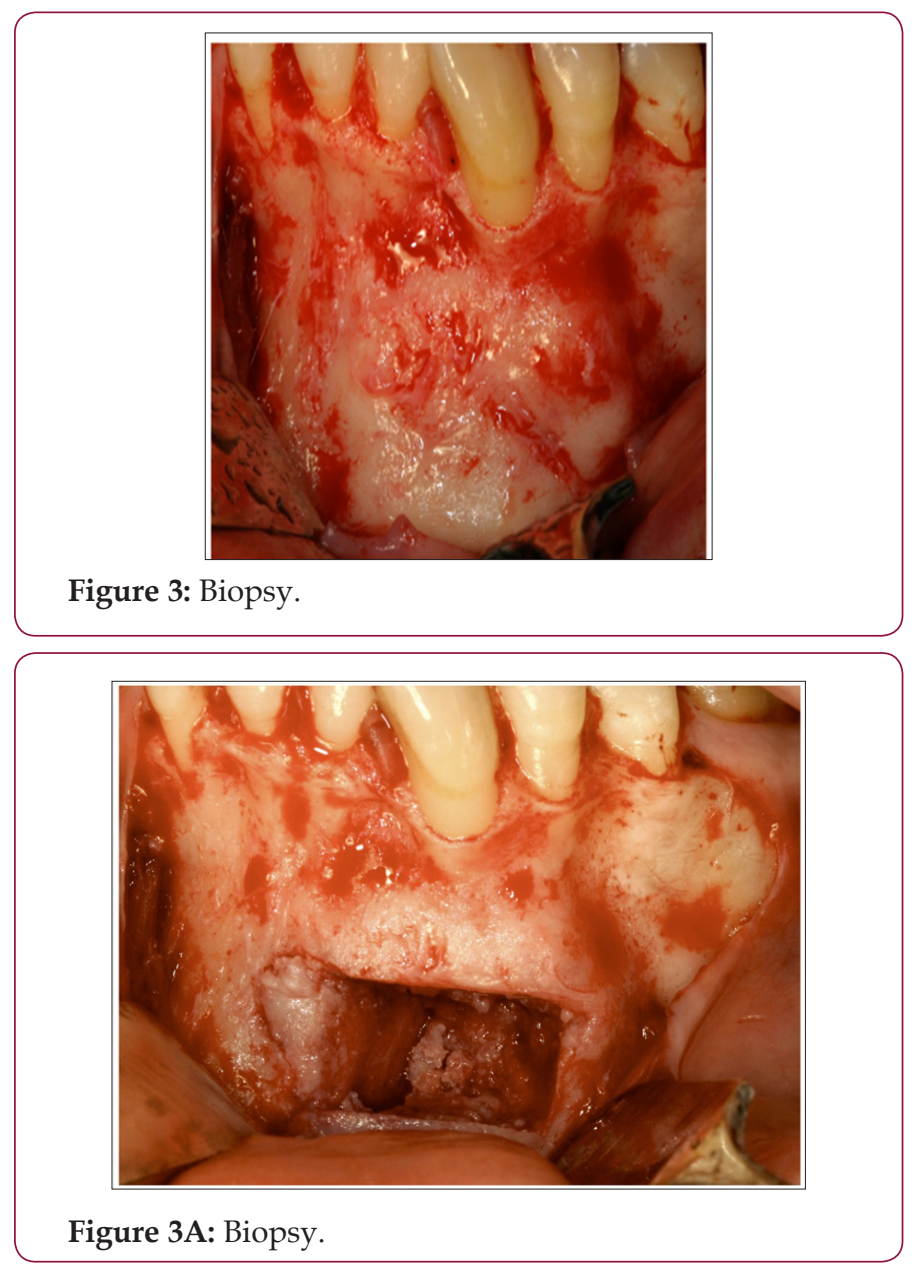

Figure 3A: Biopsy.

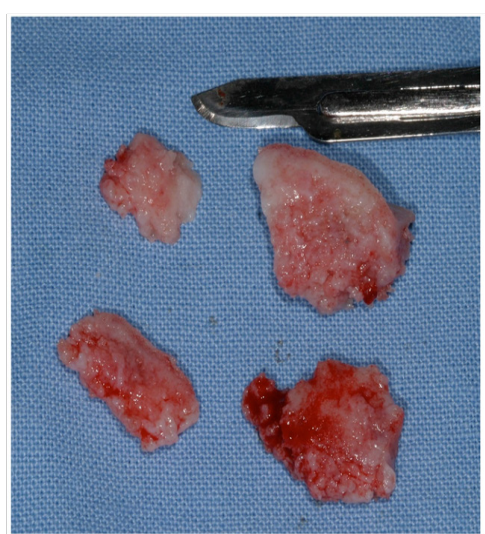

Figure 3B: Biopsy.

In October 2009, she underwent a lower jaw resection on the left side, affected by the lesion, at the Unit of Maxillofacial surgery Prof. Brusati [3-18]. At the same time the resection site has been rebuilt through an iliac crest auto graft (Figures 4 \& 4a). Despite the success of the auto graft, it was still persisting a problem about missing teeth from the lower right canine to the first left molar (Figure 5). Given the patient's young age and the need to substitute the missing teeth, it has been decided to proceed with a rehabilitation using osseointegrated implants. In January 2010, three implants have been placed (Bego®Semados); following the elevation of a mucoperiosteal flap with a crestal incision (Figure 6), the drilling preparation for implant osteotomy sites have been prepared with the help of a surgical guide. The right position of the implants has been verified through alignment pin (Figure 7); three implants have been inserted having a diameter of 3.75 or $4 \mathrm{~mm}$, and length of $13 \mathrm{~mm}$ (Figure 8 ). The flap was accurately sutured with 4-0 silk (Figure 9).

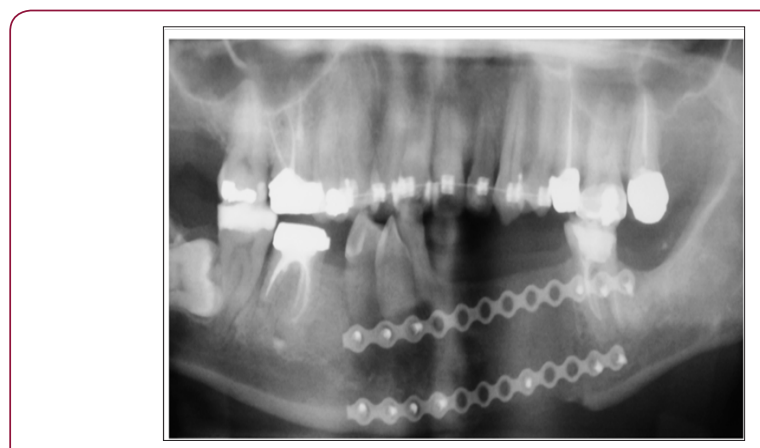

Figure 4: Iliac crest autograft.

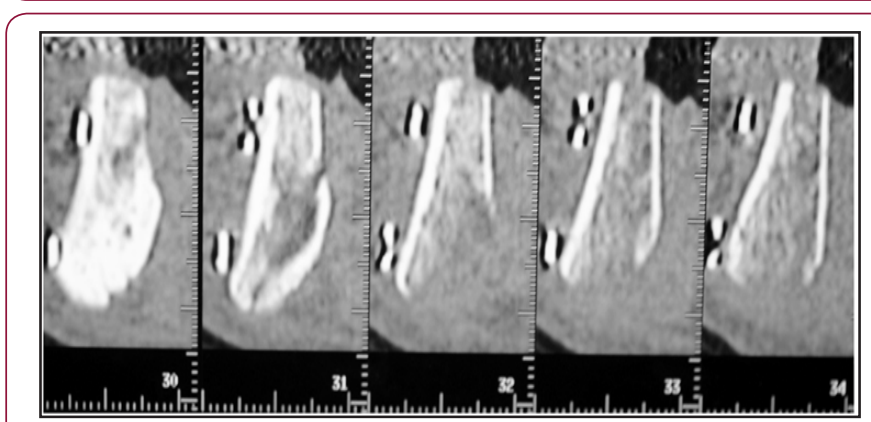

Figure 4A: Iliac crest autograft. 


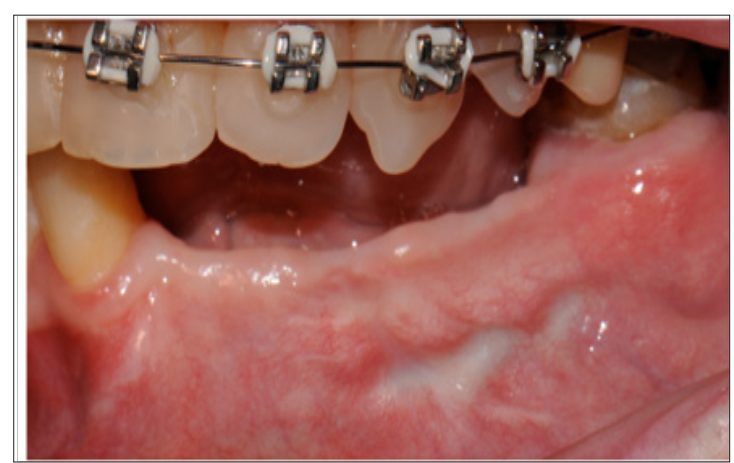

Figure 5: Post reconstruction.

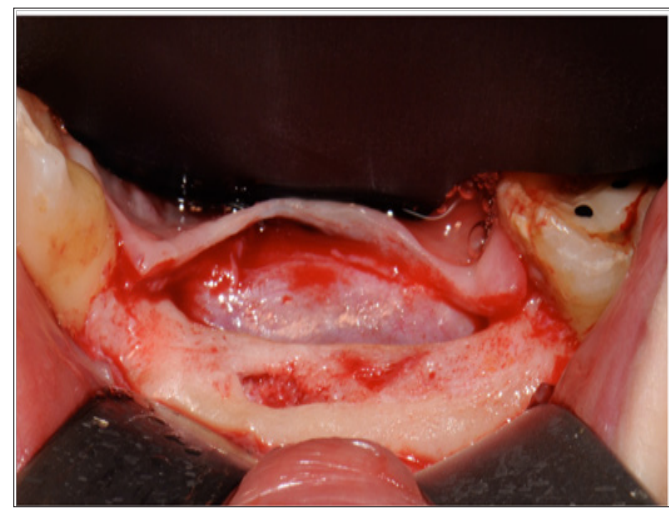

Figure 6: Crestal incision.

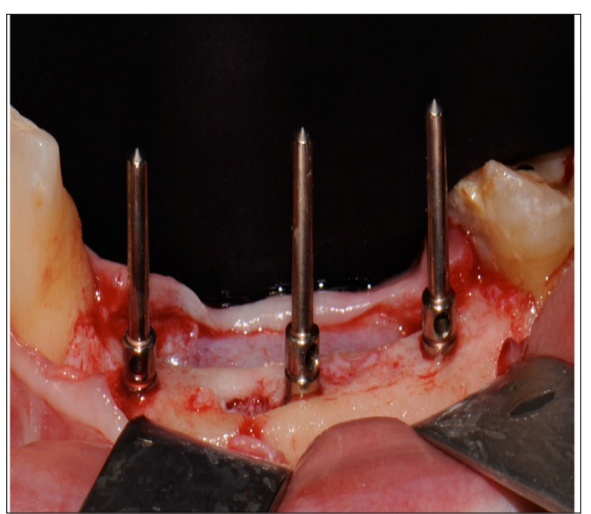

Figure 7: Implants placement.

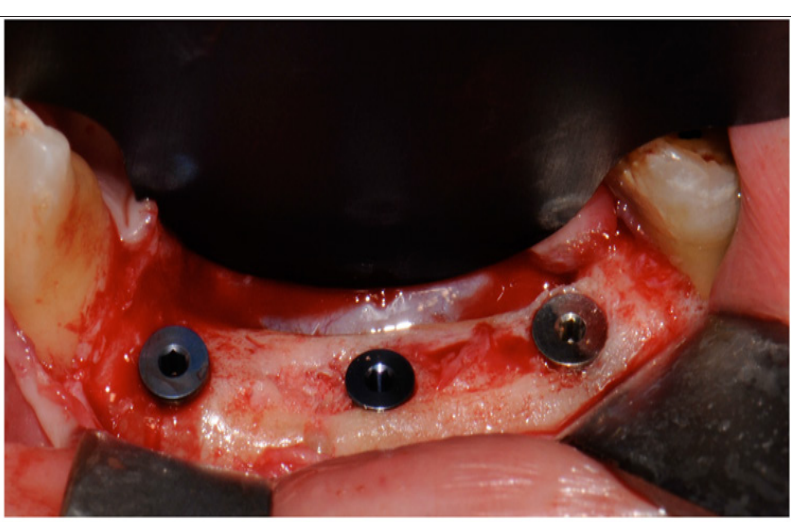

Figure 8: Implants placement.

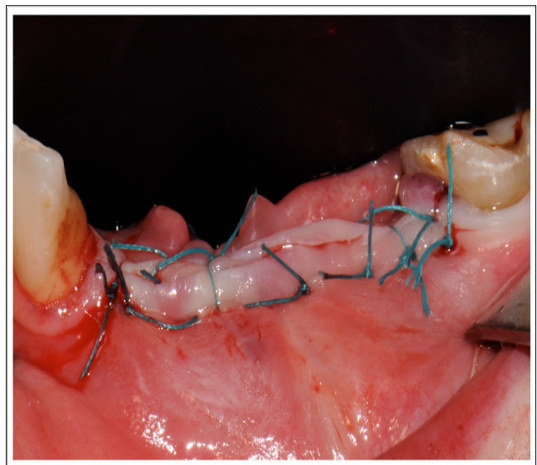

Figure 9: Suture.

The radiographic follow-up showed that the implants have been positioned correctly (Figure 10). Implant abutment connection was performed 12 weeks after implant placement, and prosthetic loading of implants was performed after 15 weeks. 8 weeks after the prosthetic loading of the implants, the cranial osteosynthesis plate has been removed with local anesthetic, practicing two incisions in the plate's attachment zones and extracting the plate from the soft tissues right after (Figures 11 \& 11b). 12 weeks later the prosthetic rehabilitation was completed using an implant fixed prosthesis (Figures 12 \& 13).

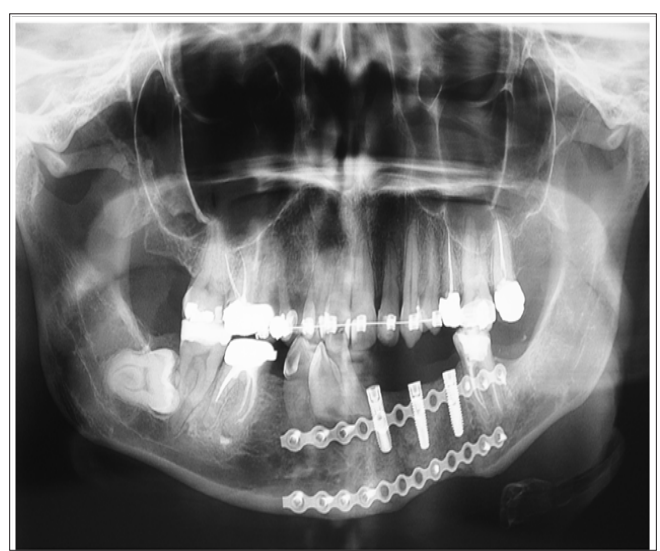

Figure 10: Radiographic follow-up.

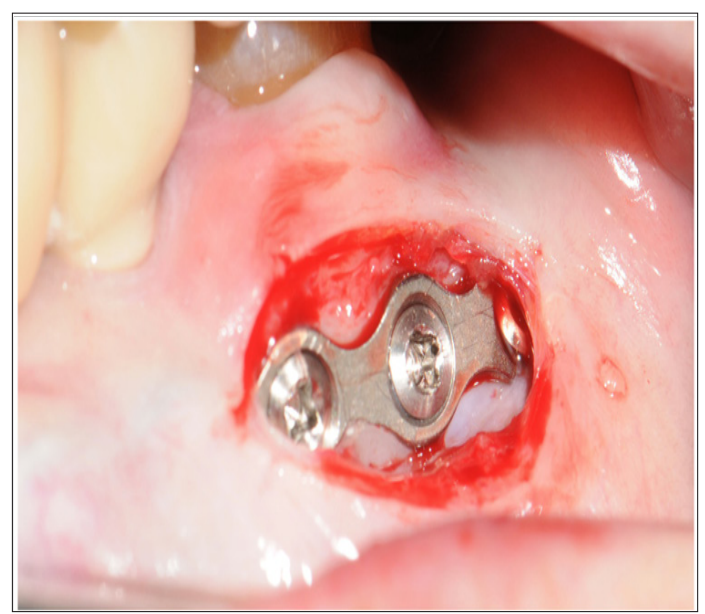

Figure 11: plate removal. 

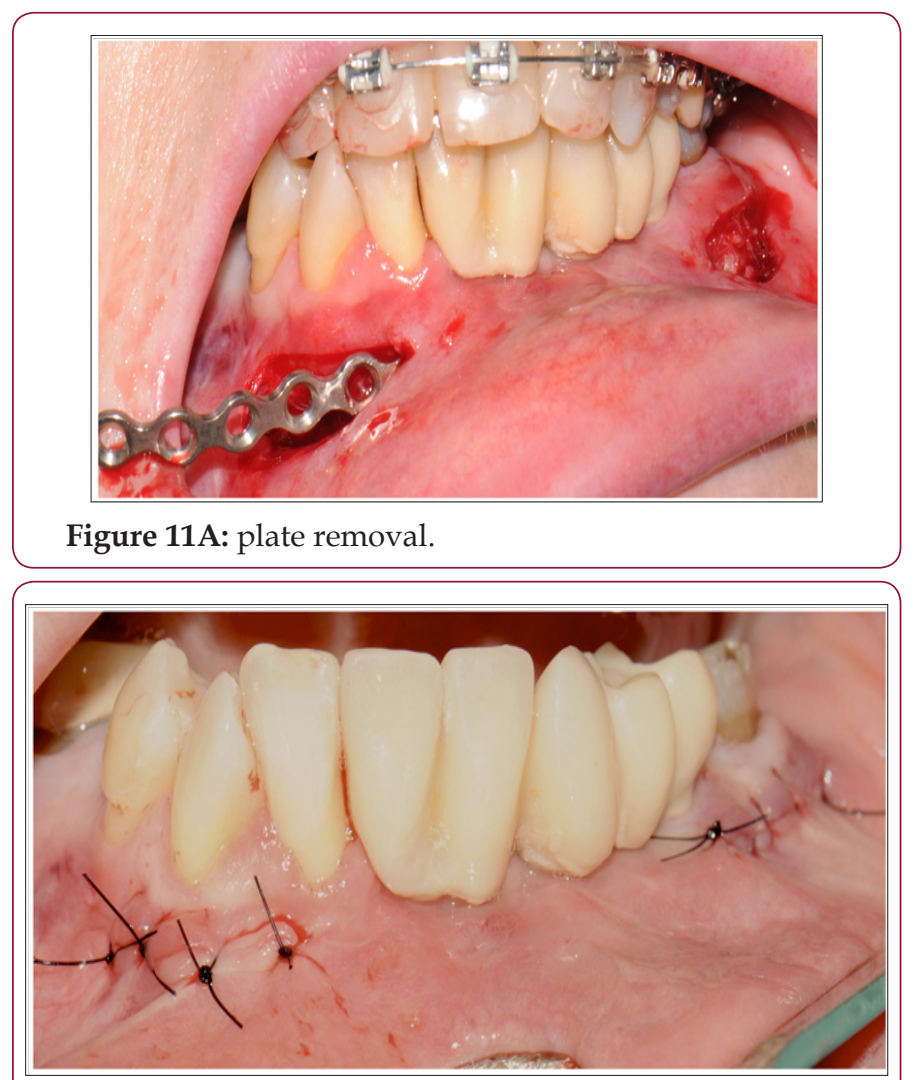

Figure 11B: plate removal.

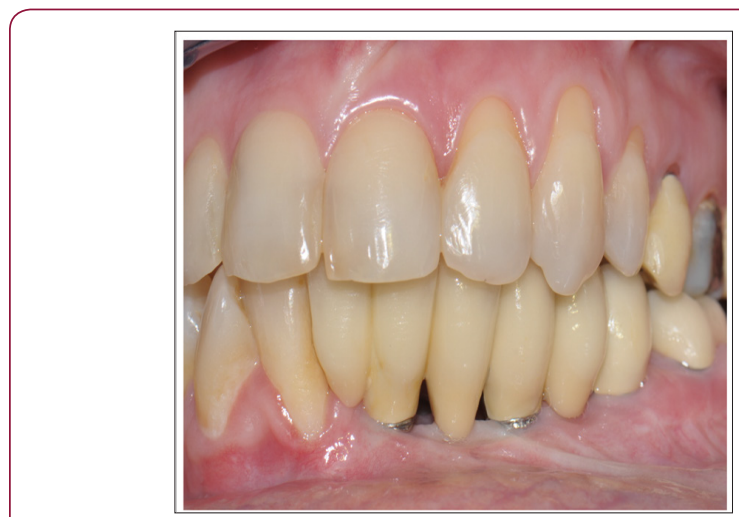

Figure 12: rosthetic rehabilitation.

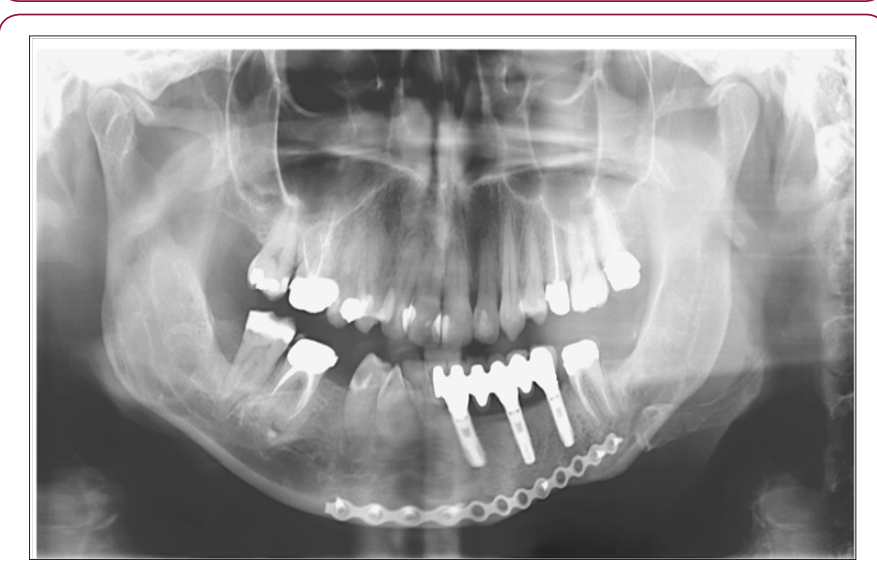

Figure 13: Prosthetic rehabilitation.

\section{Results}

The aesthetic and functional result was overall satisfying and, 18 months later the radiographic follow-up shows a stable prosthesis and the implants don't show any sign and/or symptoms of failure according to the Albrektsson parameters (Figure $14 \& 14 \mathrm{~b}$ ).

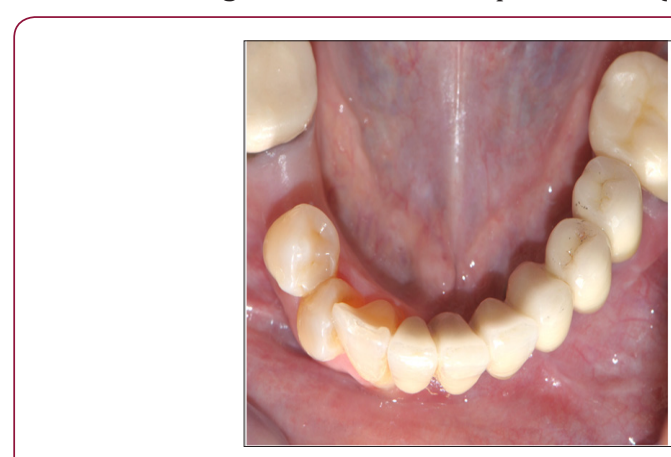

Figure 14: 18 month's follow-up.

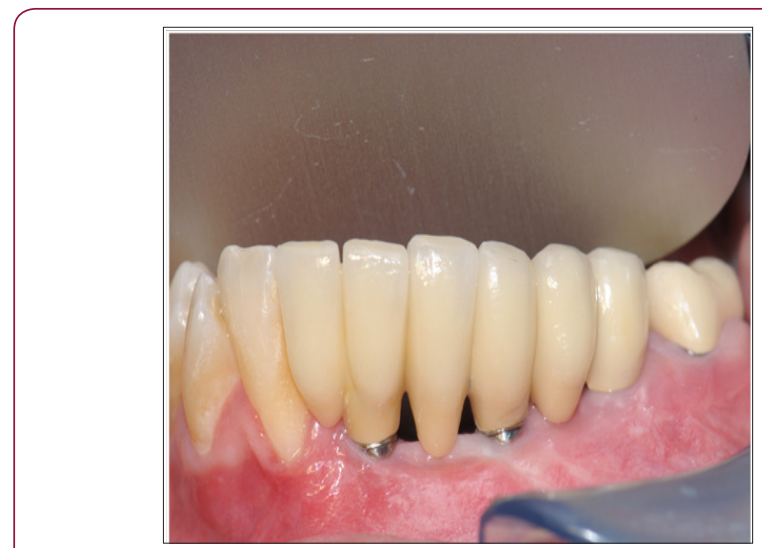

Figure 14A: 18 month's follow-up.

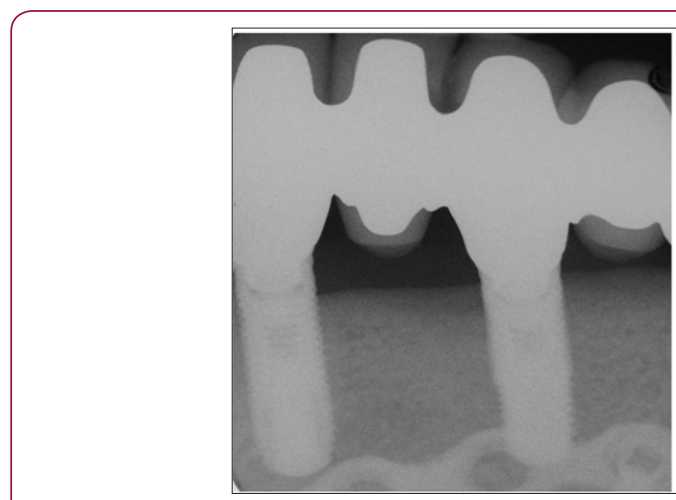

Figure 14B: 18 month's follow-up.

\section{Discussion}

The resective surgical treatment of maxillofacial neoplasms determines a series of craniofacial functional and aesthetic alterations that are often hard to fix. Therefore, new functional and aesthetic reconstruction methods are proposed (rigid fixation with titanium plate, titanium mesh with bone chips, bone auto graft, free graft). All these techniques allow restoring bone continuity, and determining a notable functional and aesthetic improvement; but only using 
bone grafts or bone chips it's possible proceed with a rehabilitation using osseointegrated implants. This kind of implants allows the substitution of the missing teeth and provides a functional rehabilitation of the edentulous areas. Previously the rehabilitation of this site was performed by dentures, local anatomy of edentulous alveolar ridges may not be suitable in the resection site and it compromises the chewing stability.

The use of osseointegrated implants and grafts technique has become one of the main rehabilitation methods for these patients, with predictable long-term results. The implant survival rate values obtained in bone grafts are similar to those found in literature for implants placed in native bone. Usually rehabilitation following the malignant neoplasm surgical resection is powered by free graft and free flap; while the rehabilitation following benign bone neoplasm surgical resection is performed by auto graft when the soft tissues are preserved. Today, the most performed reconstruction methods are iliac crest graft and calvarial bone graft. The iliac crest graft allows obtaining both cortical bone and spongy bone; instead, calvarial bone graft allows for obtaining only cortical bone. The iliac crest graft is ideal for rehabilitation after jaw resection because it allows a tridimensional reconstruction; the calvarial bone graft is the best choices in case of a residual bony floor where the graft can be fixed. The calvarial bone graft is thin so it's necessary to use a layer technique in order to obtain a correct vertical augmentation. The osteointegration of the two grafts has different time schedules; in fact, it is determined by our embryological origin. Nowadays before the implant placement it's recommended to wait 3-4 month after iliac crest graft, and 5-6 month after calvarial bone graft. Generally it is suggested to wait time of 6 month for both the grafts because there is an high risk of higher bone loss during the prosthetic rehabilitation. Moreover the delayed insertion of implants allows the disease follow up, the patient evaluation after the surgery, and a correct rehabilitation planning with surgical mask. The success of implants rehabilitation depends on the selection of the patient; in fact, it's important to consider according to inclusion criteria the neoplasm benignity. The literature suggests using implants rehabilitation only in some specific cases: acceptable vertical intermaxillary relationship, enough tongue function, neoplasm good prognosis, soft tissues health. The implant sites are prepared as usually but some precautions are needed. The periostal elevation has to be minimized in order to preserve the vascularity of graft and reduce the possibilities of bone seizure. In particular the surgical reconstruction procedure adopted by maxillofacial surgery department Prof. Brusati [3] was performed with 2 osteosynthesis plates. The caudal osteosynthesis plate is stabilized with osteosynthesis screws that are inserted both in the proximal and distal bone graft, and in the residual bone. The superior osteosynthesis plate is stabilized only with osteosynthesis screws inserted in the residual bone. The lack of osteosynthesis screws in the reconstruction area represents an advantage because it allows implants placement with preservative flap. It's not necessary to expose the graft to remove the screws; moreover the cranial osteosynthesis plate has been removed practicing two incisions in the screw zones and extracting the plate from the soft tissues right after. So the periostal elevation over the iliac crest graft was minimized during the implants placement and it reduced the risk of higher bone loss.

\section{Conclusion}

The case report, as described in literature, shows that the functional and aesthetic rehabilitation after resective surgical treatment of neoplasm using bone graft and implants placement, is a rehabilitation methods that leads to predictable long-term results. Survival rate of implants placed in this patient are consistent with those reported in the literature for implants placed in native, non reconstructed bone.

\section{Acknowledgment}

We would like to thank Prof. Roberto Brusati head of the Unit of Maxillofacial surgery, Department of Health Sciences, Santi Paolo \& Carlo Hospital, University of Milan, Milan, Italy for the surgical reconstruction of the treated patient.

\section{References}

1. Von Recklinghausen FD (1891) Die Fibrose oder deformierende ostitis, die osteomalacie, und die ostoplastiche carcinose in ihren gegenselt-gen beziehungen. Berlin Festschr, Rudolf Virchow.

2. Chen YR, Noordhoff MS (1990) Treatment of cranimaxillofacial fibrous dysplasia: how early and how extensive? Plast Reconstr Surg 86(5): 835-842.

3. Brusati R, Chiapasco M (1999) Elementi di chirurgia oromaxillo-facciale. Ed. Masson.

4. Ozek C, Gundogan H, Bilkay U, Tokat C, Gurler T, et al. (2002) Craniomaxillofacial fibrous dysplasia. JCraniofac Surg. 13(3): 382-389.

5. Ficcara G (2006) Manuale di patologia e medicina orale. Ed. McGraw-Hill.

6. Ricalde P,Horsewell BB (2001) Craniofacial fibrous dysplasia of the fronto orbital region. A case series and literature review. J Oral Maxillofac Surg 59(2): 157-168

7. Chen Y, Noordhoff MS, Samuel M (1990) Treatment of craniomaxillofacial fibrous dysplasia: How early and how extensive? Plast Recostr Surg 86: 835-844.

8. Posnick JC, (1998) Fibrous dysplasia of the craniomaxillofacial region: Current clinical perspectives. Br J Oral Maxillofac Surg. 36(4): 264-274.

9. Zenn RM, Zuniga J (2001) Treatment of fibrous dysplasia of the mandible with radical excision and immediate reconstruction: Case report. J Craniofac Surg 12(3): 259-263.

10.Valentini V, Cassoni A, Marianetti TM, Terenzi V, Fadda MT, et al. (2009) Craniomaxillofacial fibrous dysplasia: conservative treatment or radical surgery? A retrospettive study on 68 patients. Plast Reconstr Surg 123(2): 653-660.

11.Chiapasco M, Abati S, Ramundo G, Rossi A, Romeo E, et al. (2000) Behavior of implants in bone grafts or free flaps after tumor resection. Clin Oral Implants Res 11(1): 66-75.

12.Buchbinder D, Urken M, Vickery C, Weinberg H, Sheiner A, et al. (1989) Functional mandibular reconstruction in patients with oral cancer. Oral Surg Oral Med Oral Pathol 68(4): 499-504. 
13.Brogniez V, Lejuste P, Pecheur A, Reychler H (1998) Dental prosthetic reconstruction of osseointegrated implants placed in irradiated bone. Int J Oral Maxillofac Implants 13(4): 506-512.

14.Gurlek A, Miller MJ, Jacob RF, Lively JA, Schusterman MA (1998) Functional results of dental restoration with osseointegrated implants after mandible reconstruction. Plast Reconstr Surg 101(3): 650-659.

15.Keller EE, Tolman D, Eckert S (1998) Endosseous implant and autogenous bone graft reconstruction of mandibular discontinuity: a 12-year longitudinal study of 31 patients. Int J Oral Maxillofac Implants 13(6): 767-780.
16.Sclaroff A, Haugey B, Gay WD, Paniello R (1994) Immediate mandibular reconstruction and placement of dental implants. At the time of ablative surgery. Oral Surg Oral Med Oral Pathol 78(6): 711-717.

17.Weischer T, Schettler S, Mohr C (1996) Concept of surgical and implant-supported prostheses in the rehabilitation of patients with oral cancer. Int J Oral Maxillofac Implants 11(6): 775-781.

18.Tidstrom KD, Keller EE (1990) Reconstruction of mandibular discontinuity with autogenous iliac bone graft: Report of 34 consecutive patients. Journal of Oral and Maxillofacial Surgery 48(4): 336-346.

\begin{tabular}{l} 
Assets of Publishing with us \\
$\begin{array}{l}\text { BIOMEDICAL } \\
\text { RESEARCHES }\end{array}$ \\
- Global archiving of articles \\
\hline Immediate, unrestricted online access \\
\hline
\end{tabular}

